\title{
Hepatitis B virus $X$ protein activates Notch signaling by its effects on Notch1 and Notch4 in human hepatocellular carcinoma
}

\author{
JUAN GAO, YIMIN XIONG, YAN WANG, YIMING WANG, GUORONG ZHENG and HUALIN XU \\ Department of Digestive Diseases, Wuhan General Hospital of Guangzhou Command PLA, Wuhan, Hubei 430070, P.R. China
}

Received August 17, 2015; Accepted October 1, 2015

DOI: 10.3892/ijo.2015.3221

\begin{abstract}
Deregulated expression of Notch receptors and abnormal activity of Notch signaling have been observed in a growing number of malignant tumors, however, the expression and activity of Notch in hepatitis B virus (HBV)-associated hepatocellular carcinoma (HCC) and their relationship with HBV X protein (HBx) are still not fully elucidated. To address this, we examined the overall expression of Notch receptors in HBV-associated HCC tissues, analyzed their relationship with $\mathrm{HBx}$, and further investigated the role of Notch signaling in HBx stable transfected HepG2 cells (HepG2X). The results showed that Notch signaling could be activated by $\mathrm{HBx}$ in HepG 2 cells. The expression of cytoplasmic Notch1 or nuclear Notch4 was correlated with the expression of $\mathrm{HBx}$ in HBV-associated HCC tissues. The expression of cytoplasmic Notch1 or nuclear Notch4 could also be upregulated by HBx in HepG2X cells. The upregulation of Notch1 by HBx was through p38 MAPK pathway. Moreover, HBx was found to directly interact with Notch1, whereas, not with Notch4 in HepG2X cells. Suppression of Notch signaling by $\gamma$-secretase inhibitor (GSI) decreased cell growth, blocked cell cycle progression and induced cell apoptosis in HepG2X cells. The present study indicates that $\mathrm{HBx}$ activates Notch signaling by its effects on Notch1 and Notch4, and therefore, recruits Notch signaling as a downstream pathway contributing to its carcinogenic role in $\mathrm{HBV}$-associated HCC.
\end{abstract}

\section{Introduction}

Notch signaling controls various biological events and is vital for many types of cell fate determination in embryonic development. Four Notch receptors (Notch1-4) and two groups of ligands, Jagged and Delta-like, have been identified in mammals (1). The activation of Notch signaling is initialized by the binding of Notch receptors and ligands, which leads to

Correspondence to: Dr Juan Gao, Department of Digestive Diseases, Wuhan General Hospital of Guangzhou Command PLA, 627 Wuluo Road, Wuhan, Hubei 430070, P.R. China

E-mail: gaojuanwh@hotmail.com

Key words: hepatocellular carcinoma, hepatitis B virus $\mathrm{X}$ protein, Notch signaling, Notch1, Notch4 the release of intracellular region of Notch (ICN) from Notch transmembrane subunit (NTM) and then translocation to the nucleus to activate transcription of a group of downstream genes (2). Recent studies have revealed the deregulation of expression and function of Notch receptors and ligands in a series of malignant tumors. The expression of Notch1 and Jagged1 are upregulated in human colon adenocarcinoma (3). Notch1, Notch2, and hairy and enhancer of split 1 (HES1) show high expression in biliary tract cancer (4). Notch3 has a stronger positive degree of expression in lung squamous cell carcinoma and adenocarcinoma compared with the corresponding non-tumor tissue (5). The expression of Notch1 and Jagged 1 in both metastatic prostate cancer and high grade prostate cancer is significantly higher than those in low grade prostate cancer and in benign prostatic tissues (6). Besides, Notch2 and Jagged1 expression levels correlate with survival of colorectal cancer patients (7), and nuclear expression of Notch1 and Notch3 is also found to be associated with tumor recurrence in colorectal cancer (8). Overexpression of Notch1 is also identified as a significant and independent prognostic factor for esophageal squamous cell cancer (9).

HBV is a major cause of HCC, and HBx has been reported as an important viral protein in the carcinogenesis and progression of HBV-associated HCC (10). Studies show that $\mathrm{HBx}$ interacts with transcription factors in the nucleus and activates signal transduction cascades in the cytoplasm $(11,12)$, which in the end induces foci in cells and leads to liver cancer in transgenic mice $(13,14)$. There have been some studies on the relationship between $\mathrm{HBx}$ and Notch. Researchers from a Chinese laboratory reported that $\mathrm{HBx}$ promotes the growth of human hepatic L02 and HCC HepG2 cells via the activated Notch pathway, and Notch1 is a potential therapeutic target for the treatment of HBx-associated HCC (15-17). However, this same team also published a study reporting that $\mathrm{HBx}$ downregulates $\mathrm{NF}-\kappa \mathrm{B}$ signaling through decreasing Notch signaling pathway in L02 cells (18), which seems the opposite to their previous results and is therefore puzzling. Besides, there was still another study showing that HBx decreases ICN1 in HCC Huh7 cells by reducing Notch1 cleavage, which results in enhanced cell proliferation (19). These results are inconsistent, and we noted that they are all based on cell research and data from tissue samples is lacking.

Therefore, it is very necessary to investigate the overall expression of Notch receptors and their relationship with $\mathrm{HBx}$ in HBV-associated HCC tissues. In the present study, 
we examined the expression and activity of Notch in both $\mathrm{HBV}$-associated HCC tissues and HBx expressed HCC cells and found that HBx activated Notch signaling by its effects on Notch1 and Notch4 in HBV-associated HCC.

\section{Materials and methods}

Human tissue samples, cell lines and drugs. HCC tissue samples were obtained from 44 patients with HBV infection who received surgical resection at Xijing Hospital of the Fourth Military Medical University from 2002 to 2004 with informed consents of the the patients and with the approval of the Human Research Committee of the University and Wuhan General Hospital. pcDNA3-HBx was constructed by inserting a wild-type HBx cDNA fragment into pcDNA3 (Invitrogen, Carlsbad, CA, USA). pcDNA3 stable transfected HepG2 cells (HepG2-pc) and HepG2X cells were established in our perious study (20). HeLa cells were purchased from the American Type Culture Collection (ATCC; Manassas, VA, USA). For drug treatment, HepG2-pc and HepG2X cells were treated with the following drugs for $24 \mathrm{~h}$ at different final concentrations $(5,10,20$ and $40 \mu \mathrm{M})$ to inhibit the activity of MEK, PI-3K and p38 MAPK: PD98059 (Calbiochem, Darmstadt, Germany), LY294002 (Calbiochem), SB203580 (Alexis Biochemicals, San Diego, CA, USA). DMSO $(20 \mu \mathrm{M})$ was used as drug control. For suppression of Notch signaling, HepG2-pc and HepG2X cells were treated with N-[N-(3,5difluorophenacetyl-L-alanyl)]-S-phenyl glycine t-Butyl Ester (DAPT; Calbiochem) at different final concentrations (2.5, 5 and $10 \mu \mathrm{M})$. DMSO $(5 \mu \mathrm{M})$ was used as drug control.

Luciferase assay. pGa981-6, a reporter gene plasmid which contained a hexamerized 50 bp Epstein-Barr virus nuclear antigen 2 response element (EBNA2RE) of the TP1 promotor in front of the luciferase gene, was strictly dependent on RBP-J (21). pRL-TK (Promega, Madison, WI, USA) was co-transfected as an internal control for transfection efficiency. A negative control plasmid (neg-pGa981-6) was constructed by replacing EBNA2RE with an irrelevant DNA segment. For luciferase assay, pcDNA3-HBx, pGa981-6 and pRL-TK were co-transfected into HepG2 cells with Lipofectamine ${ }^{\mathrm{TM}} 2000$ (Invitrogen). DAPT at different final concentrations (2.5, 5 and $10 \mu \mathrm{M}$ ) was added to the transactivation system to suppress Notch signaling. pcDNA3 and neg-pGa981-6 replacing pcDNA3-HBx and pGa981-6, respectively, were used as negative controls. Forty-eight hours later, co-transfected cells were lysed and luciferase assays were performed in Luminometer TD-20/20 (Turner Designs, Inc., Sunnyvale, CA, USA).

Immunohistochemical staining. Horseradish peroxidase staining was used to visualize antigens on paraffin-embedded $5-\mu \mathrm{m}$ sections. Sections were incubated with anti-Notch1 primary goat polyclonal antibody (pAb), anti-Notch2, 3 and 4 primary rabbit pAb (1:100; Santa Cruz Biotechnology, Santa Cruz, CA, USA), or anti-HBx mouse monoclonal antibody (mAb) (1:50; Chemicon International, Temecula, CA, USA), and then detected with Histostain ${ }^{\mathrm{TM}}$-SP (Zymed Laboratories, San Francisco, CA, USA). In negative controls, non-immune IgGs were used to substitute for the primary antibody. All stained sections were evaluated by two independent investi- gators who were blinded to the groups. The scoring was based on intensity and the extent of the staining (22). The immunoreactive score of each section was calculated by sum of these two parameters and graded as negative (I), weak (II), moderate (III) and strong (IV).

Reverse transcription-polymerase chain reaction (RT-PCR). Total cellular RNA was extracted using the One Step RT-PCR kit (MBI, Vilnius, Lithuania). Semi-qualitative PCR was performed using human primer sequences for Notch1-4 and for $\mathrm{HBx}$ as in our previous reports $(20,22)$. $\beta$-actin was used as a housekeeping gene. Gene expression was presented by the relative yield of the PCR product from target sequences to that from $\beta$-actin gene.

Western blot analysis. Immunoblotting was performed using the anti-Notch1-4 pAbs (1:300), the anti-HBx mAb (1:100), anti-phospho-MAPKAPK-2 rabbit $\mathrm{mAb}$ (1:500; Cell Signaling Technology, Boston, MA, USA), anti-phospho-p44/42 MAPK (Erk1/2) rabbit mAb (1:1,000; Cell Signaling Technology) and anti-phospho-Akt rabbit mAb (1:500; Cell Signaling Technology) followed by incubation with peroxidase coupled anti-goat, anti-rabbit or anti-mouse $\mathrm{IgG}$, respectively. HeLa cells were used as positive control for the expression of Notch receptors. Autoradiograms were quantified by densitometry using Bio Image Intelligent Quantifier (IQ) software. Relative protein levels were calculated by referring them to the amount of $\beta$-actin protein.

Double immunofluorescence staining. HBV-associated HCC tissues and HepG2X cells were stained with anti-Notch1 goat $\mathrm{pAb}$ and anti-HBx mouse $\mathrm{mAb}$, or anti-Notch4 rabbit $\mathrm{pAb}$ and anti-HBx mouse mAb, respectively. After incubated with tetraethyl rhodamine isothiocyanate-labeled rabbit antigoat IgG (1:100; Chemicon) and fluorescein isothiocyanate (FITC)-conjugated goat anti-mouse $\operatorname{IgG}(1: 80$; Chemicon) or tetraethyl rhodamine isothiocyanate-labeled goat anti-rabbit IgG (1:100; Chemicon) and FITC-conjugated goat anti-mouse $\mathrm{IgG}$, all sections were examined by a laser scanning confocal microscope (Bio-Rad Laboratories, Hercules, CA, USA). Non-immune IgGs in substitution for the primary antibodies were considered negative controls.

Co-immunoprecipitation assay. HepG2X and HepG2-pc cells were lysed and the lysate was pretreated with Protein G Plus/Protein A Agarose (Calbiochem) to remove non-specifically bound proteins. Immunoprecipitation (IP) was carried out with anti-HBx mouse $\mathrm{mAb}$ or non-immune mouse $\mathrm{IgG}$ and the agarose beads. After incubated at $4^{\circ} \mathrm{C}$ for $4 \mathrm{~h}$, the beads were washed and subjected to western blot analysis with the anti-Notch1 pAb, anti-Notch4 pAb or anti-HBx mAb.

Cell proliferation and colony-forming assay. For 3-(4,5-dimethylthiazolyl-2)-2,5-diphenyltetrazolium bromide (MTT) assay, HepG2-pc and HepG2X cells were seeded and cultured overnight, then DAPT at different final concentrations was added and $5 \mu \mathrm{M}$ DMSO was used as drug control. After 1, 2, 3, 4, 5, 6 and 7 days of drug treatment, the cell survival rates were assessed using MTT assay. The growth inhibitory rate was calculated as: (control A value - treated A value)/ 
control A value $\mathrm{x} 100 \%$. For colony-forming assay, HepG2-pc and HepG2X cells were treated as above. After 2 weeks of incubation, cell colonies were fixed and stained with Giemsa dye and colonies containing $>50$ cells were counted. For anchorage-independent cell proliferation, 24-well plates were coated with $0.5 \%$ of bottom agar solution, and $2 \times 10^{3} \mathrm{HepG} 2-\mathrm{pc}$ and HepG2X cells were embedded into $0.3 \%$ top agar gel containing DAPT of 2.5, 5 and $10 \mu \mathrm{M}$, respectively. The dishes were examined with vertical microscope for colony formation after a 2-week incubation period. Colonies of $>75 \mathrm{~mm}$ was counted.

Propidium iodide (PI) staining and flow cytometry (FCM). HepG2-pc $\left(1 \times 10^{6}\right)$ and HepG2X cells were seeded and incubated overnight, then $5 \mu \mathrm{M}$ DAPT was added. After $24 \mathrm{~h}$ of incubation, cells were harvested, fixed and resuspended in PI solution. Samples were then analyzed for their DNA content by flow cytometer (Beckman Coulter, Inc., Brea, CA, USA).

Apoptosis assay. For Annexin V binding assay, HepG2-pc and HepG2X cells were treated with $5 \mu \mathrm{M}$ DAPT, respectively. PI and Annexin $\mathrm{V}$ were added to those cells successively, and the cells were analyzed by FCM. Mean values from three independent experiments were accepted as results. For transmission electron microscopy (TEM), HepG2-pc and HepG2X cells treated with DAPT were fixed, dehydrated and embedded. Cells were then sectioned and stained. The cell ultrastructure was assessed by TEM at 4,000-fold magnification.

Statistical analysis. Statistical analysis was performed using the SPSS software (version 13.0; SPSS, Inc., Chicago, IL, USA). Correlations between Notch receptors and $\mathrm{HBx}$ were analyzed using Spearman's rank correlation. Two-sided Student's t-test was used for comparisons. $\mathrm{P}<0.05$ was considered as statistically significant.

\section{Results}

HBx activates Notch signaling in HepG2 cells. To investigate whether Notch signaling could be activated by HBx, luciferase assay was carried out with co-transfection of pGa981-6 and pcDNA3-HBx into HepG2 cells. The data showed that HBx activated Notch signaling in a dose-dependent manner $(\mathrm{P}<0.05)$, and the activation was significantly suppressed by 5 or $10 \mu \mathrm{M}$ DAPT (Fig. 1). The control sample with pcDNA3 replacing pcDNA3-HBx showed luciferase activity to some extent, indicating the endogenous activity of Notch signaling in HepG2 cells.

HBx upregulates the expression of Notchl through $p 38$ MAPK pathway and activates Notch 4 in HBV-associated HCC tissues and HepG2X cells. Since Notch signaling could be activated by HBx, we wonder which Notch receptor is involved in the activation. For this, we examined the expression of Notch1-4 in $44 \mathrm{HBV}$-associated HCC tissues by immunohistochemical staining. The result showed that Notch1-4 were all expressed in the neoplastic cells of HCCs with different intensity and extensity. Cytoplasmic Notch1 expression was strong and could be detected in $93.2 \%$ (41/44) of HCCs. Nuclear Notch1 was weak and the positive rate was $11.4 \%(5 / 44)$. Notch2

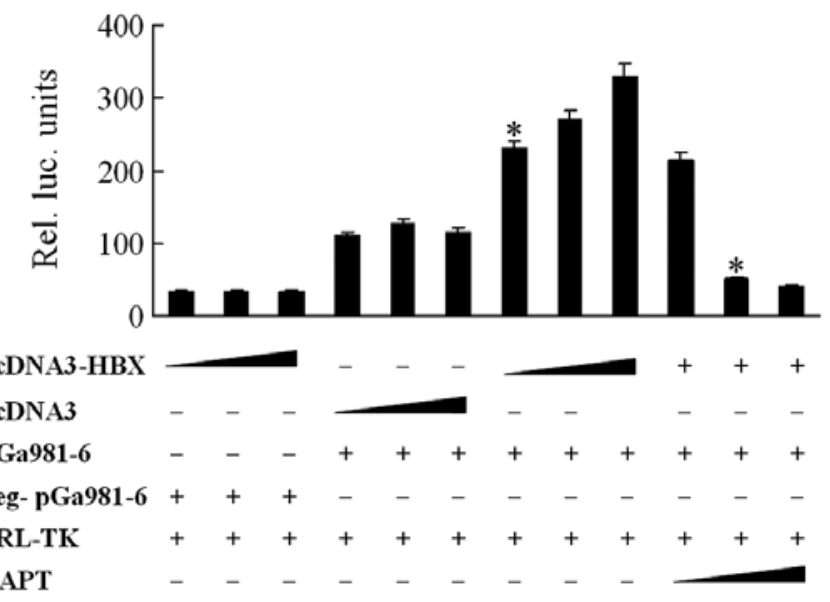

Figure 1. HBx activates Notch signaling in HepG2 cells. The activation of Notch signaling by HBx was examined by luciferase assay. ${ }^{*} \mathrm{P}<0.05$.

Table I. Expression of Notch receptors and HBx in HBVassociated HCC tissues.

\begin{tabular}{|c|c|c|c|c|c|c|}
\hline & \multirow{2}{*}{$\begin{array}{c}\text { Positive } \\
\text { cases }\end{array}$} & \multicolumn{4}{|c|}{ Score } & \multirow[b]{2}{*}{ rs } \\
\hline & & I & II & III & IV & \\
\hline Notch1 (cytoplasmic) & 41 & 3 & 10 & 26 & 5 & $0.396^{\mathrm{a}}$ \\
\hline Notch1 (nuclear) & 5 & 39 & 4 & 1 & 0 & \\
\hline Notch2 & 14 & 30 & 12 & 2 & 0 & \\
\hline Notch3 & 27 & 17 & 18 & 9 & 0 & \\
\hline Notch4 (cytoplasmic) & 33 & 11 & 15 & 17 & 1 & \\
\hline Notch4 (nuclear) & 28 & 16 & 11 & 16 & 1 & $0.407^{\mathrm{a}}$ \\
\hline $\mathrm{HBx}$ & 38 & 6 & 26 & 12 & 0 & \\
\hline
\end{tabular}

${ }^{\mathrm{a}} \mathrm{P}<0.01, \mathrm{n}=44$.

and Notch3, detected only in the cytoplasm, were expressed in $31.8 \%(14 / 44)$ and $61.4 \%$ (27/44) of HCCs, respectively. Cytoplasmic and nuclear expression of Notch4 was detected in $75.0 \%(33 / 44)$ and $63.6 \%(28 / 44)$ of HCCs, respectively. HBx immunoreactivity was observed in $86.4 \%$ (38/44) of HCCs, with positive signal in the cytoplasm (Fig. 2A). Spearman analysis showed that there was a significant correlation between HBx and cytoplasmic Notch1 or nuclear Notch4 immunoreactivity, with $\mathrm{rs}=0.396, \mathrm{P}<0.01$ (HBx and Notch1), and rs $=0.407, \mathrm{P}<0.01$ (HBx and Notch4), whereas other Notch molecules and HBx did not show such a relationship (Table I).

The expression of Notch molecules was further examined in HepG2X cells. RT-PCR showed that the mRNA level of Notch1 was much higher in HepG2X than that in HepG2-pc cells $(\mathrm{P}<0.05)$. Notch2, Notch3 and Notch4 mRNA showed no difference between the two cell lines (Fig. 2B). For the protein level, the result of the western blot analysis showed that the proteins of Notch1-4 were all expressed in HepG2-pc and HepG2X cells, with specific bands at $~ 70-120 \mathrm{kDa}$. Notch1, Notch 2 and Notch 3 showed only one band, which was the Notch transmembrane subunit (NTM) form (23). The expression of Notch1 was higher in HepG2X than in HepG2-pc 
A

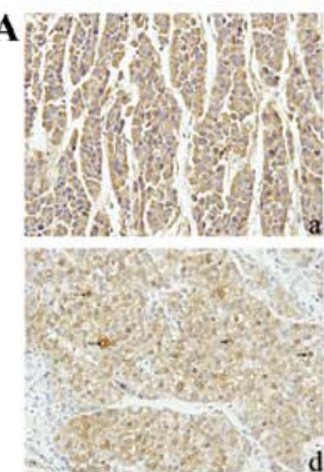

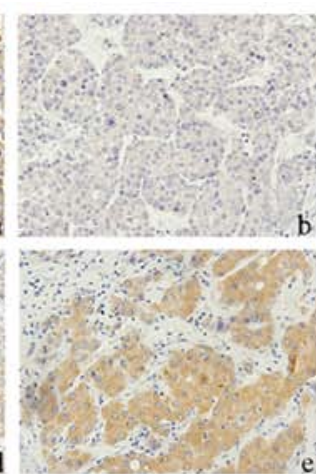

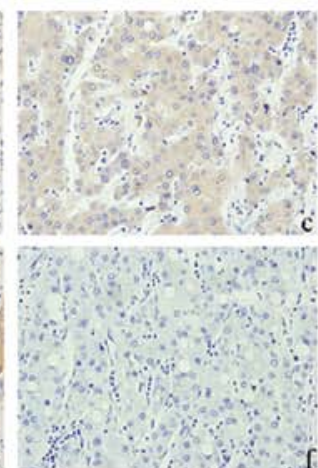

C
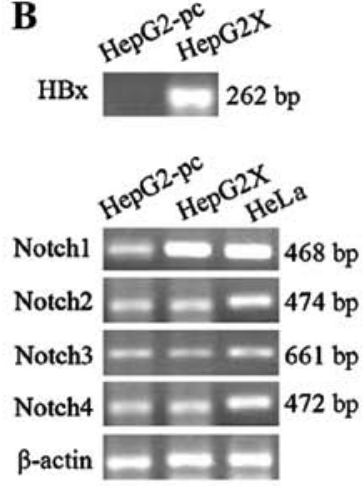

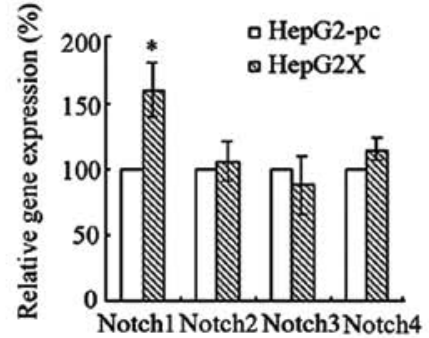

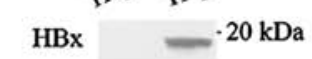

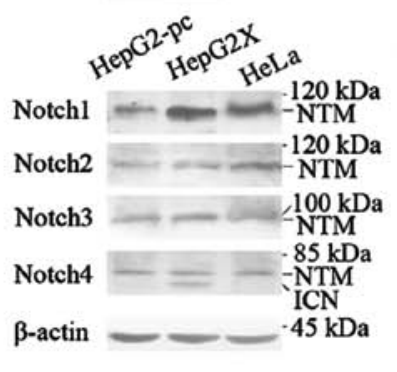

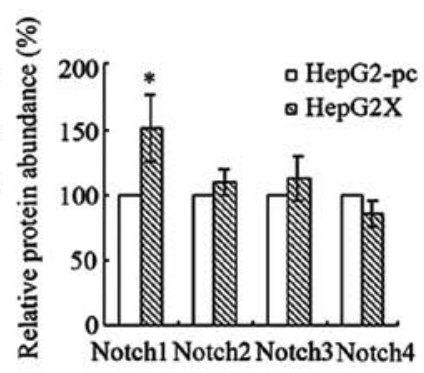

D
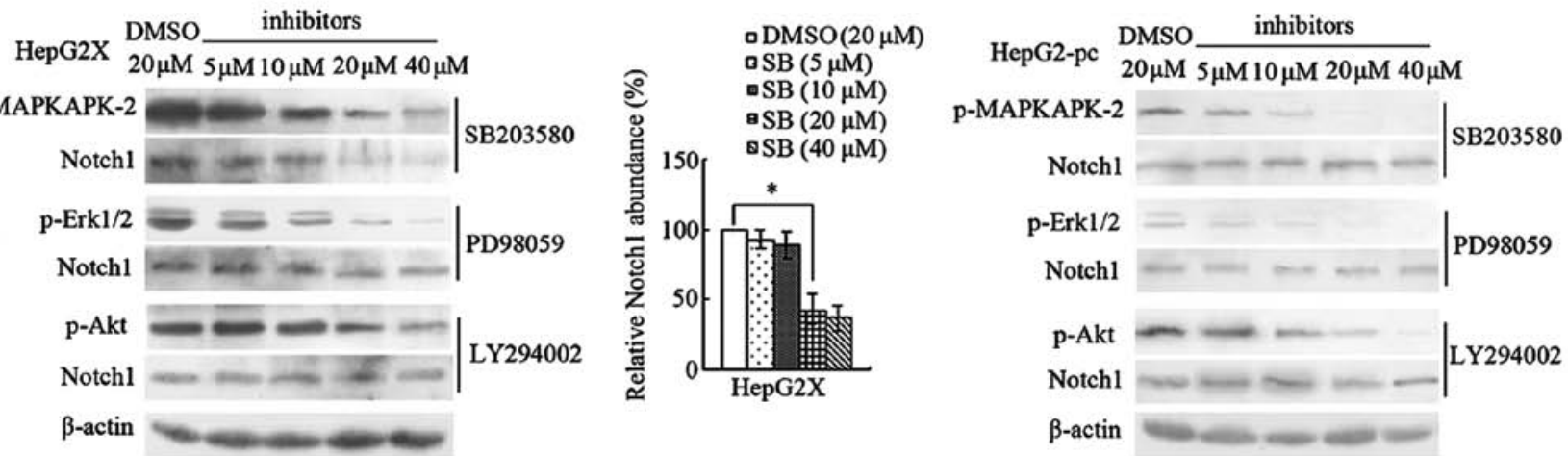

Figure 2. HBx upregulates the expression of Notch1 through p38 MAPK and activates Notch4 in HBV-associated HCC tissues and HBx expressed HepG2 cells. (A) The expression of Notch1-4 and HBx in HBV-associated HCC tissue sections were detected by immunohistochemistry. The images depict representative examples showing expression of (a) Notch1, (b) Notch2, (c) Notch3, (d) Notch4 and (e) HBx. (f) The negative control (magnification, x200). (B) Amplified products of HBx and Notch1-4 from representative RT-PCR are shown. (C) Protein levels of HBx and Notch1-4 were examined by western blot analysis. Autoradiograms were quantified by densitometry and relative mRNA and protein levels were calculated by referring them to the amount of $\beta$-actin mRNA and protein. Data were normalized by setting the ratios of the mRNA and protein levels of Notch1 to Notch4 against $\beta$-actin in HepG2-pc cells at 100\%. (D) Regulation of Notch1 by HBx through p38 MAPK pathway. Data was normalized by setting the ratio of the protein level of Notch1 against $\beta$-actin in HepG2X cells treated with $20 \mu \mathrm{M}$ DMSO at $100 \%$. $^{*} \mathrm{P}<0.05$.

cells $(\mathrm{P}<0.05)$. Notch2 and Notch3 were equally expressed between the two cell lines. Notch4 showed two bands, one of which with a higher molecular mass was its NTM form, and the lower one was its intracellular region of Notch (ICN) form (24). Although the NTM form of Notch4 was equally expressed between HepG2X and HepG2-pc cells, the ICN form appeared only in HepG2X (Fig. 2C).

Since HBx activates Ras/Raf/MEK, PI-3K/Akt and p38 MAPK pathways (25-27), and MAPK and p38 signaling can upregulate the expression of various Notch molecules in certain context $(28,29)$, we wonder whether the regulation of Notch1 by HBx is also mediated by these pathways. For this, inhibitors of p38, MEK and PI-3K were used to treat HepG2X and HepG2-pc cells, and the expression of their target as well as Notch1 was examined. The result showed that $10 \mu \mathrm{M}$ SB203580, $5 \mu \mathrm{M}$ PD98059 and $20 \mu \mathrm{M}$ LY294002 began to inhibit phospho-MAPKAPK-2, phospho-Erk1/2 and phosphoAkt in HepG2X cells, respectively, and only SB203580 decreased the expression of Notch1 (NTM) $(\mathrm{P}<0.05)$. In HepG2-pc cells, although $5 \mu \mathrm{M}$ SB203580, $5 \mu \mathrm{M}$ PD98059 and $10 \mu \mathrm{M}$ LY294002 began to suppress their targets respectively, none of them could influence the expression of Notch1 (Fig. 2D). These results demonstrated that HBx upregulated Notch1 and activated Notch4 in HBV-associated HCC, and the upregulation of Notch1 by HBx was through p38 MAPK pathway. 
A

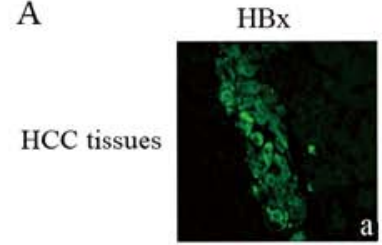

HepG2X cells
Notch1
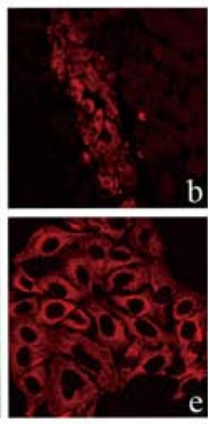

merge
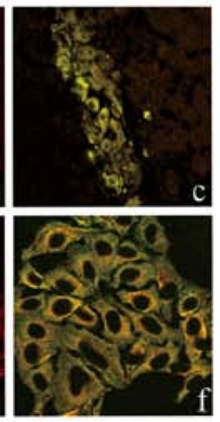
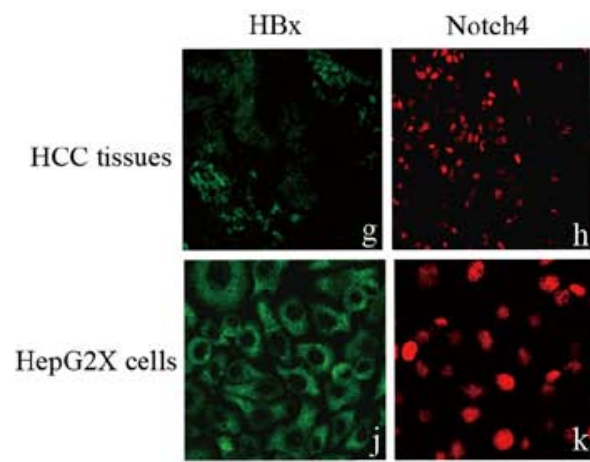

merge

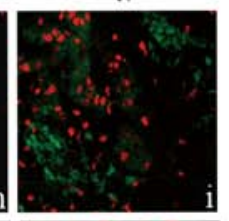

B
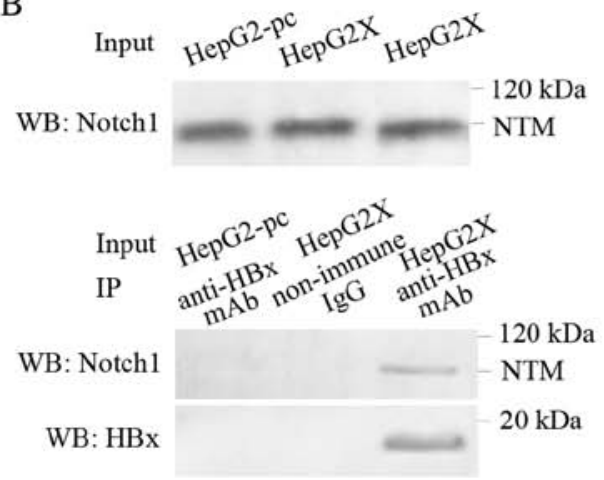

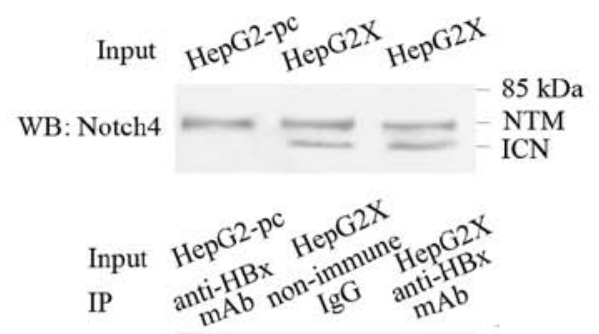

WB: Notch4

WB: HBx
$20 \mathrm{kDa}$

Figure 3. HBx morphologically co-localizes and physically interacts with Notch1 in HBV-associated HCC tissues and HepG2X cells. (A) The co-localization of Notch1 or Notch4 with HBx was examined by double immunofluorescence staining. HBx was stained green in HCC tissues (a and g) and HepG2X cells ( $d$ and j). Notch1 and Notch4 were stained red in HCC tissues (b and h) and HepG2X cells (e and k), respectively. Results of dual-labeling experiments of Notch1 and HBx are shown in HBV-associated HCC tissues (c) and HepG2X cells (f), respectively. Results of dual-labeling experiments of Notch4 and HBx are shown in HBV-associated HCC tissues (i) and HepG2X cells (1), respectively (magnification, x400). (B) The physical interaction of Notch1 or Notch4 with HBx was examined by co-immunoprecipitation assay. Western blot analysis for Notch1 and Notch4 from input and for HBx after IP showed equal amount of input protein and immunoprecipitated protein.

Notch1 morphologically co-localizes and physically interacts with $H B x$. The relationship of Notch1, Notch4 with HBx suggested a possible co-localization, and we explored this possibility by confocal microscopy. As the result showed, HBx was stained green and Notch1 was stained red. The yellow staining in dual-labeling experiments indicated overlapping areas of red and green fluorescent labels and suggested co-localization of Notch1 with HBx in the cytoplasm of neoplastic cells of HBV-associated HCC tissues and HepG2X cells. For HBx and Notch4, no apparent yellow fluorescent was observed in dual-labeling experiments (Fig. 3A). No fluorescent showed up in negative control sections. Confocal analysis demonstrated that Notch1, but not Notch4, morphologically co-localized with HBx.

We further examined the possible physical interaction of HBx with these two Notch receptors in HepG2X cells by co-immunoprecipitation assay. The result showed that Notch1 (NTM) was co-immunoprecipitated with HBx in HepG2X cells. No interaction was observed between Notch4 and HBx. No interaction was observed in HepG2-pc cells. In addition, no non-specific interaction was observed from control non-immune IgG, indicating the specificity of Notch1-HBx interaction (Fig. 3B).

Suppression of Notch signaling in HepG2X inhibits cell growth and blocks cell cycle progression. To investigate the effects of the activation of Notch signaling by $\mathrm{HBx}$ on HCC, the activity of Notch signaling in HepG2X and HepG2-pc cells was suppressed by a highly specific GSI, DAPT and cell growth was examined. The result from MTT showed that the inhibition of DAPT on the growth of HepG2X cells were observed at the final concentrations (5 and $10 \mu \mathrm{M}$ ) for 1 day $(\mathrm{P}<0.05)$, and the degree of inhibition was positively correlated with exposure time. The inhibition effect of $10 \mu \mathrm{M}$ was similar with that of $5 \mu \mathrm{M}$ DAPT, which indicated $5 \mu \mathrm{M}$ a suitable concentration (Fig. 4A). The result of colony-formation assay and soft agar assay also showed that 5 or $10 \mu \mathrm{M}$ DAPT while not DMSO significantly reduced the capacity of single cell colony formation and anchorage-independent cell proliferation in HepG2X cells $(\mathrm{P}<0.05)$. Cell growth and colony formation in HepG2-pc was not influenced significantly by DAPT (Fig. 4B and C). For cell cycle, PI staining and FCM analysis demonstrated a significant increase in the number of cells in G1 phase after HepG2X cells was treated with $5 \mu \mathrm{M}$ DAPT while not $5 \mu \mathrm{M}$ DMSO $(\mathrm{P}<0.05)$. Neither DAPT nor DMSO influenced HepG2-pc cell cycle distribution (Fig. 4D). The above results showed that suppression of Notch signaling in HepG2X inhibited cell growth and proliferation, and blocked cell cycle progression.

Suppression of Notch signaling in HepG2X induces cell apoptosis. Cell apoptosis was further examined. Apoptosis 

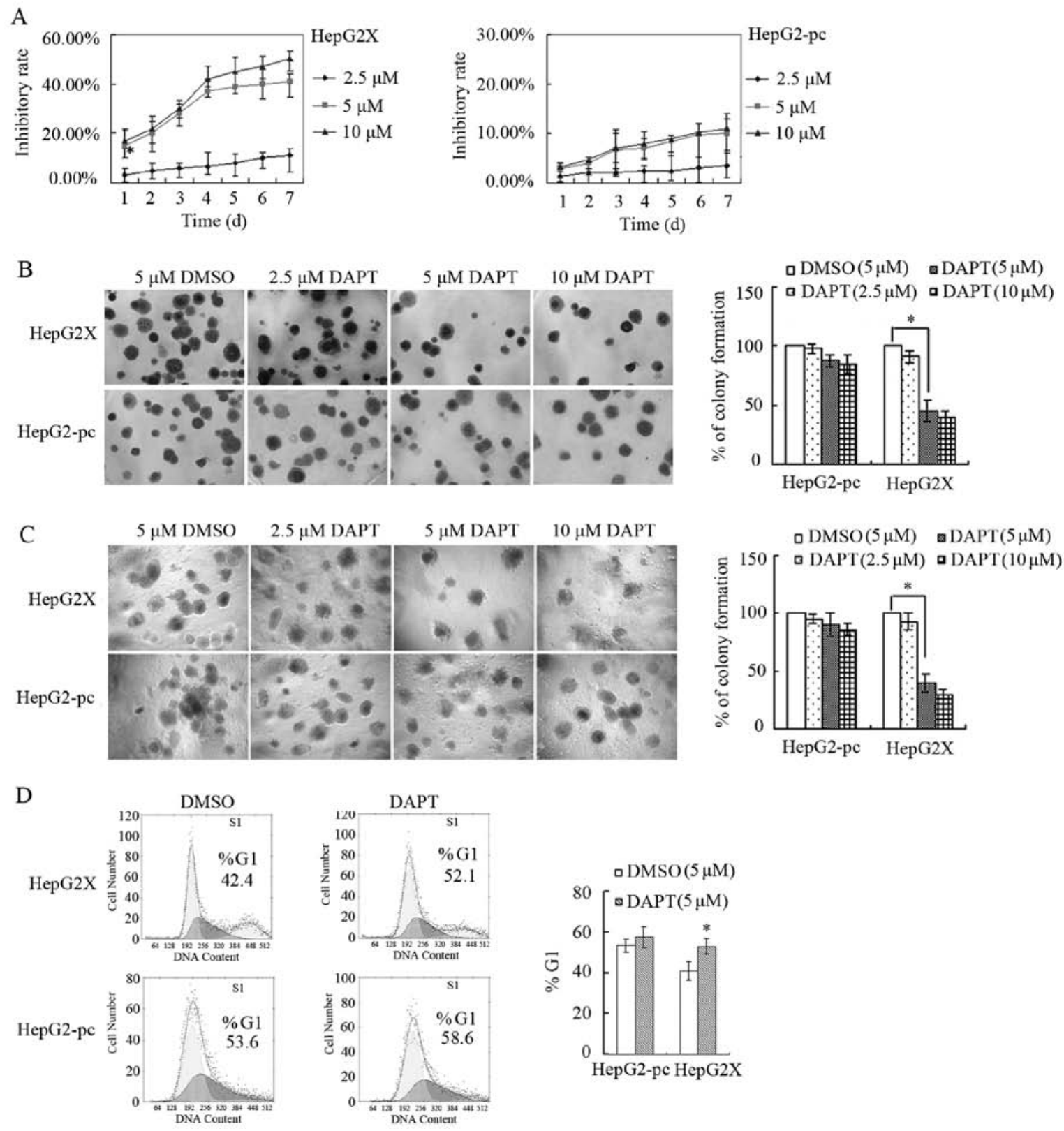

Figure 4. Suppression of activity of Notch signaling in HepG2X inhibits cell growth and blocks cell cycle progression. (A) MTT assay. (B) Colony-formation assay. (C) Soft agar assay. Data were normalized by setting the number of cell colonies in HepG2X or HepG2-pc cells treated with $5 \mu$ M DMSO at 100 . The results are given in percentages. (D) Cell cycle analysis shown by DNA histograms. ${ }^{*} \mathrm{P}<0.05$.

histograms from PI staining showed that the percentage of apoptotic cells in HepG2X dramatically increased to $23.4 \%$ after treated with DAPT, compared to $0.98 \%$ when treated with DMSO ( $\mathrm{P}<0.01)$ (Fig. 5A). Apoptosis was also assessed by Annexin $\mathrm{V}$ and PI double-staining assay. The percentage of apoptotic cells (Annexin $\mathrm{V}^{+} / \mathrm{PI}$, early stage; Annexin $\mathrm{V}^{+} / \mathrm{PI}^{+}$, late stage) of HepG2X treated with DMSO was $2.3 \%$. After treatment with $5 \mu \mathrm{M}$ DAPT for $24 \mathrm{~h}$, the percentages of apoptotic cells significantly increased to $19.1 \%(\mathrm{P}<0.01)$ (Fig. 5B). We also examined ultrastructure of cell apoptosis by TEM. DAPT $(5 \mu \mathrm{M})$ led to a characteristic pattern of apoptosis in HepG2X cells. No such characteristic apoptosis pattern was observed in the DMSO control (Fig. 5C). In all the above assays, DAPT did not induce apparent cell apoptosis in HepG2-pc. These results demonstrated that suppression of activity of Notch signaling could lead to cell apoptosis in HepG2X cells.

\section{Discussion}

The role of Notch in HCC has been studied comprehensively. Our previous research demonstrated that Notch1 activation contributes to HCC cell growth and proliferation (30). We also found that Jagged1 is highly expressed in HCC tissues and its expression is closely related with HBx (20). Afterwards, studies from other research teams showed that downregulation of Notch1 inhibits the invasion and migration of HCC cells by inactivating the cyclooxygenase-2/Snail/E-cadherin pathway, and also by regulation of phosphatase and tensin homolog (PTEN) and focal adhesion kinase (FAK) $(31,32)$. 
A
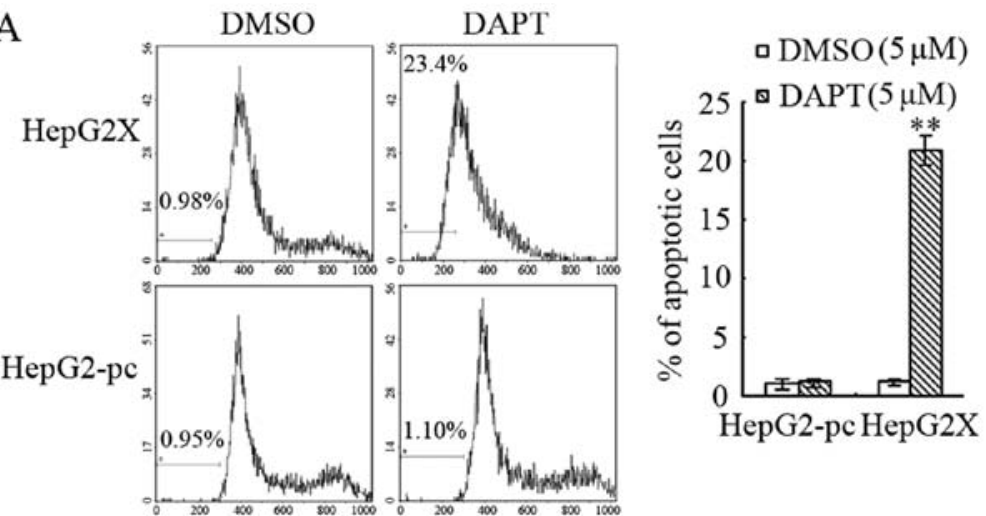

B
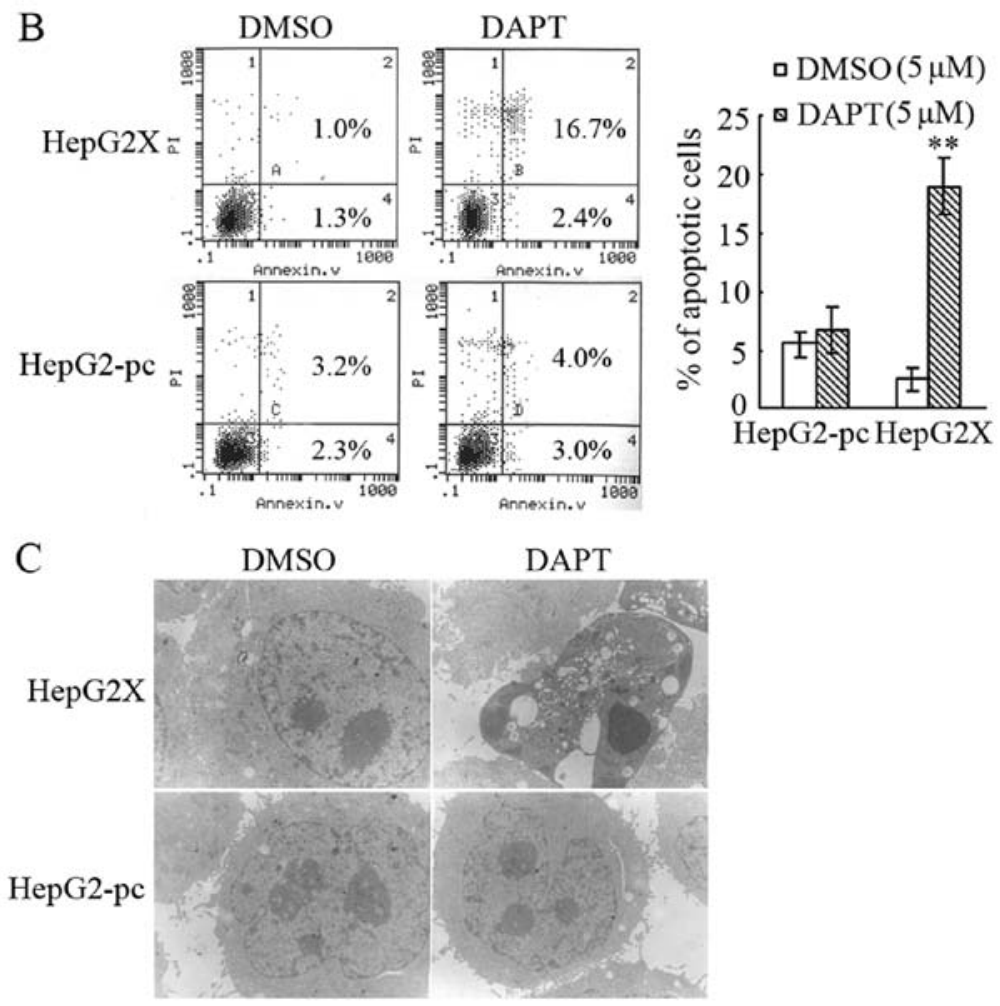

Figure 5. Suppression of activity of Notch signaling induces apoptosis in HepG2X cells. (A) Representative apoptosis histograms from PI staining and FCM analysis. (B) Annexin V binding assay. Data were normalized by setting the number of apoptotic cells in HepG2X or HepG2-pc cells treated with $5 \mu$ M DMSO at 100 . The results are given as percentage; ${ }^{* *} \mathrm{P}<0.01$. (C) TEM scanning. DAPT induced characteristic changes of apoptosis in HepG2X cells, with chromosome condensation and aggregation, nuclear fragmentation, cytoplasmic condensation, blebbing and emergence of apoptotic bodies (magnification, $\mathrm{x} 4,000)$.

There are also some studies investigating the role of Notch in the prognosis of HCC patients. Researchers found that patients with high Notch1 or Notch3 expression are at a significantly increased risk for shortened survival time in HCC patients (33). Both Notch1 expression and Notch4 overexpression are independent predictors of shorter disease-specific survival in HCC (34). These reports indicate an oncogenic role of Notch in $\mathrm{HCC}$.

HBx has been reported to have an important effect on the carcinogenesis and progression of HBV-associated $\mathrm{HCC}$ by its regulation of a series of signal pathways and molecules and thus also affects signal transduction and biological behavior of the host cells. Although HBx has been inferred as an important carcinogenic factor, more vital downstream effectors remain to be identified. Since Notch signaling has been demonstrated to play an oncogenic role in HCC, we studied its relationship with $\mathrm{HBx}$ and investigated its role in HBV-associated HCC. Our results show that HBx activates Notch signaling in a dosedependent manner. To explore which Notch receptor is involved in the activation, we examined the expression of Notch1-4 in HBV-associated HCC tissues and HepG2X cells. The results show that the level of Notch1 (NTM) is upregulated by HBx. The inhibitor of p38 MAPK blocks the increase of Notch1 by HBx, which indicates that HBx regulates Notch1 through the p38 MAPK pathway. Moreover, ICN4 is found to accumulate in HBV-associated HCC tissues and HepG2X cells. Since ICN is rapidly degraded after it activates transcription in the nucleus, the accumulation of ICN4 in our case is obviously due to the activation of Notch 4 by HBx. Therefore, we conclude that HBx activates Notch signaling by its activation of Notch4.

Notch1 was found in a very small number of nuclear positive samples in HBV-associated HCC tissues and did not detect 
its activated form in HepG2X cells, which seems to indicate that it is the expression, not the activity, of Notch1 which is regulated by $\mathrm{HBx}$. However, there is still another possible reason for the undetectable ICN1, that is, the extremely poor stability of ICN1 in our case. It has been found that ICN1 is degraded in the nucleus by Fbw7 E3 ligase-mediating ubiquitination (35). Upon Notch receptor-ligand binding, Jagged1 ligand undergoes a proteolytic cleavage, resulting in the production of a free intracellular domain (JICD), which is reported to reduce the stability of ICN1 through Fbw7mediating ubiquitination and degradation of the protein (36). In fact, Jagged 1 was found to be upregulated by HBx in our previous experiment (20). Thus, the undetectability of ICN1 in the present study might be due to the increased JICD, which still needs further investigation.

The relationship of Notch1, Notch4 with HBx indicates a possible physical interaction between them. Actually, we find that Notch1, but not Notch4, morphologically co-localizes and physically interacts with $\mathrm{HBx}$. Our result demonstrates that, besides regulation of expression, the effects of $\mathrm{HBx}$ on Notch1 might also result from their interaction. HBx has been reported to interact with various proteins to take part in the carcinogenesis of $\mathrm{HCC}(37,38)$. The interaction of HBx with Notch1 in our case might lead to activation of Notch signaling or other crosstalk signaling pathways, which would probably contribute to the development of HBV-associated HCC.

Furthermore, we investigated the role of the activated Notch signaling in HepG2X cells. After suppression of Notch signaling, cell growth and proliferation of HepG2X is significantly attenuated, while no apparent growth inhibition occurs in the control HepG2-pc cells with a lower activity of Notch signaling. Moreover, we found that suppression of Notch signaling induces cell cycle arrest and cell apoptosis in HepG2X cells. Thus, it can be concluded that HBx promotes cell growth and inhibits cell apoptosis in HCC through its activation of Notch signaling. Previous reports showed that Notch signaling can be activated by several viral oncogenes, such as EBNA2 and human papillomavirus E6 and E7 proteins, and take part in the carcinogenesis of these virus-associated cancers $(39,40)$. Thus, our finding indicates Notch signaling as a downstream pathway activated by $\mathrm{HBx}$ in $\mathrm{HBV}$-associated HCC.

In conclusion, the present study indicates that $\mathrm{HBx}$ activates Notch signaling by its effects on Notch1 and Notch4 and, therefore, recruits Notch signaling as a downstream pathway contributing to its carcinogenic role in HBV-associated HCC.

\section{Acknowledgements}

The authors gratefully acknowledge the Department of Pathology (Xijing Hospital, the Fourth Military Medical University) for providing HCC tissue samples. The present study is supported by the Military Medical Scientific Youth Cultivation Project (no. 13QNP053) and the Wuhan Young and Middle-aged Medical Backbone Personnel Training Project.

\section{References}

1. Artavanis-Tsakonas S, Rand MD and Lake RJ: Notch signaling: Cell fate control and signal integration in development. Science 284: 770-776, 1999.
2. Davis RL and Turner DL: Vertebrate hairy and Enhancer of split related proteins: Transcriptional repressors regulating cellular differentiation and embryonic patterning. Oncogene 20: 8342-8357, 2001

3. Gao J, Liu J, Fan D, Xu H, Xiong Y, Wang Y, Xu W, Wang Y, Cheng Y and Zheng G: Up-regulated expression of Notch1 and Jagged1 in human colon adenocarcinoma. Pathol Biol (Paris) 59: 298-302, 2011.

4. Mazur PK, Riener MO, Jochum W, Kristiansen G, Weber A, Schmid RM and Siveke JT: Expression and clinicopathological significance of notch signaling and cell-fate genes in biliary tract cancer. Am J Gastroenterol 107: 126-135, 2012.

5. Zhou M, Jin WY, Fan ZW and Han RC: Analysis of the expression of the Notch3 receptor protein in adult lung cancer. Oncol Lett 5: 499-504, 2013.

6. Zhu H, Zhou X, Redfield S, Lewin J and Miele L: Elevated Jagged-1 and Notch-1 expression in high grade and metastatic prostate cancers. Am J Transl Res 5: 368-378, 2013.

7. Jin HY, Zhang HY, Wang X, Xu J and Ding Y: Expression and clinical significance of Notch signaling genes in colorectal cancer. Tumour Biol 33: 817-824, 2012.

8. Ozawa T, Kazama S, Akiyoshi T, Murono K, Yoneyama S, Tanaka T, Tanaka J, Kiyomatsu T, Kawai K, Nozawa H, et al: Nuclear Notch3 expression is associated with tumor recurrence in patients with stage II and III colorectal cancer. Ann Surg Oncol 21: 2650-2658, 2014.

9. Ogawa R, Ishiguro H, Kimura M, Funahashi H, Wakasugi T, Ando T, Shiozaki M and Takeyama H: NOTCH1 expression predicts patient prognosis in esophageal squamous cell cancer. Eur Surg Res 51: 101-107, 2013.

10. Tiollais P, Pourcel C and Dejean A: The hepatitis B virus. Nature 317: 489-495, 1985.

11. Maguire HF, Hoeffler JP and Siddiqui A: HBV X protein alters the DNA binding specificity of CREB and ATF-2 by proteinprotein interactions. Science 252: 842-844, 1991.

12. Kekulé AS, Lauer U, Weiss L, Luber B and Hofschneider PH: Hepatitis B virus transactivator HBx uses a tumour promoter signalling pathway. Nature 361: 742-745, 1993.

13. Kim CM, Koike K, Saito I, Miyamura T and Jay G: HBx gene of hepatitis B virus induces liver cancer in transgenic mice. Nature 351: 317-320, 1991

14. Shirakata Y, Kawada M, Fujiki Y, Sano H, Oda M, Yaginuma K, Kobayashi $\mathrm{M}$ and Koike $\mathrm{K}$ : The $\mathrm{X}$ gene of hepatitis $\mathrm{B}$ virus induced growth stimulation and tumorigenic transformation of mouse NIH3T3 cells. Jpn J Cancer Res 80: 617-621, 1989.

15. Wang F, Zhou H, Yang Y, Xia X, Sun Q, Luo J and Cheng B: Hepatitis $B$ virus $X$ protein promotes the growth of hepatocellular carcinoma by modulation of the Notch signaling pathway. Oncol Rep 27: 1170-1176, 2012.

16. Wang F, Xia X, Wang J, Sun Q, Luo J and Cheng B: Notch1 signaling contributes to the oncogenic effect of HBx on human hepatic cells. Biotechnol Lett 35: 29-37, 2013.

17. Sun Q, Wang R, Wang Y, Luo J, Wang P and Cheng B: Notch1 is a potential therapeutic target for the treatment of human hepatitis B virus X protein-associated HCC. Oncol Rep 31: 933-939, 2014.

18. Luo J, Zhou H, Wang F, Xia X, Sun Q, Wang R and Cheng B: The hepatitis $B$ virus $X$ protein downregulates NF- $\kappa$ B signaling pathways through decreasing the Notch signaling pathway in HBx-transformed L02 cells. Int J Oncol 42: 1636-1643, 2013.

19. Xu J, Yun X, Jiang J, Wei Y, Wu Y, Zhang W, Liu Y, Wang W, Wen Y and Gu J: Hepatitis B virus X protein blunts senescencelike growth arrest of human hepatocellular carcinoma by reducing Notch1 cleavage. Hepatology 52: 142-154, 2010.

20. Gao J, Chen C, Hong L, Wang J, Du Y, Song J, Shao X, Zhang J, Han H, Liu J, et al: Expression of Jagged 1 and its association with hepatitis B virus X protein in hepatocellular carcinoma. Biochem Biophys Res Commun 356: 341-347, 2007.

21. Kato H, Taniguchi Y, Kurooka H, Minoguchi S, Sakai T, Nomura-Okazaki S, Tamura K and Honjo T: Involvement of RBP-J in biological functions of mouse Notch1 and its derivatives. Development 124: 4133-4141, 1997.

22. Gao J, Song Z, Chen Y, Xia L, Wang J, Fan R, Du R, Zhang F, Hong L, Song J, et al: Deregulated expression of Notch receptors in human hepatocellular carcinoma. Dig Liver Dis 40: 114-121, 2008.

23. Struhl G and Adachi A: Nuclear access and action of notch in vivo. Cell 93: 649-660, 1998.

24. Das I, Craig C, Funahashi Y, Jung KM, Kim TW, Byers R, Weng AP, Kutok JL, Aster JC and Kitajewski J: Notch oncoproteins depend on gamma-secretase/presenilin activity for processing and function. J Biol Chem 279: 30771-30780, 2004. 
25. Benn J and Schneider RJ: Hepatitis B virus HBx protein activates Ras-GTP complex formation and establishes a Ras, Raf, MAP kinase signaling cascade. Proc Natl Acad Sci USA 91: 10350-10354, 1994.

26. Shih WL, Kuo ML, Chuang SE, Cheng AL and Doong SL: Hepatitis B virus $X$ protein activates a survival signaling by linking SRC to phosphatidylinositol 3-kinase. J Biol Chem 278: 31807-31813, 2003.

27. Tarn C, Zou L, Hullinger RL and Andrisani OM: Hepatitis B virus $X$ protein activates the p38 mitogen-activated protein kinase pathway in dedifferentiated hepatocytes. J Virol 76 9763-9772, 2002.

28. Zeng Q, Li S, Chepeha DB, Giordano TJ, Li J, Zhang H, Polverini PJ, Nor J, Kitajewski J and Wang CY: Crosstalk between tumor and endothelial cells promotes tumor angiogenesis by MAPK activation of Notch signaling. Cancer Cell 8 : $13-23,2005$

29. Weijzen S, Rizzo P, Braid M, Vaishnav R, Jonkheer SM,Zlobin A, Osborne BA, Gottipati S, Aster JC, Hahn WC, et al: Activation of Notch-1 signaling maintains the neoplastic phenotype in human Ras-transformed cells. Nat Med 8: 979-986, 2002.

30. Gao J, Dong Y, Zhang B, Xiong Y, Xu W, Cheng Y, Dai M, Yu Z $\mathrm{Xu} \mathrm{H}$ and Zheng G: Notch1 activation contributes to tumor cell growth and proliferation in human hepatocellular carcinoma HepG2 and SMMC7721 cells. Int J Oncol 41: 1773-1781, 2012.

31. Zhou L, Wang D, Li Q, Sun W, Zhang Y and Dou K: The downregulation of Notch1 inhibits the invasion and migration of HCC cells by inactivating the cyclooxygenase- $2 /$ Snail/E-cadherin pathway in vitro. Dig Dis Sci 58: 1016-1025, 2013.

32. Hu Y, Li H, Qiu K, Li D, Zhou J, Hu Y and Zhang F: Downregulation of Notch1 inhibits the invasion of human $\mathrm{HCC}$ HepG2 and MHCC97H cells through the regulation of PTEN and FAK. Int J Mol Med 34: 1081-1086, 2014.
33. Zhou L, Zhang N, Song W, You N, Li Q, Sun W, Zhang Y, Wang D and Dou K: The significance of Notch1 compared with Notch3 in high metastasis and poor overall survival in HCC. PLoS One 8: e57382, 2013

34. Ahn S, Hyeon J and Park CK: Notch1 and Notch4 are markers for poor prognosis of HCC. Hepatobiliary Pancreat Dis Int 12: 286-294, 2013

35. Oberg C, Li J, Pauley A, Wolf E, Gurney M and Lendahl U: The Notch intracellular domain is ubiquitinated and negatively regulated by the mammalian Sel-10 homolog. J Biol Chem 276: 35847-35853, 2001

36. Kim MY, Jung J, Mo JS, Ann EJ, Ahn JS, Yoon JH and Park HS: The intracellular domain of Jagged-1 interacts with Notch1 intracellular domain and promotes its degradation through Fbw7 E3 ligase. Exp Cell Res 317: 2438-2446, 2011.

37. Choi YH, Kim HI, Seong JK, Yu DY, Cho H, Lee MO, Lee JM, Ahn YH, Kim SJ and Park JH: Hepatitis B virus X protein modulates peroxisome proliferator-activated receptor gamma through protein-protein interaction. FEBS Lett 557: 73-80, 2004.

38. Kalra $\mathrm{N}$ and Kumar V: The $\mathrm{X}$ protein of hepatitis $\mathrm{B}$ virus binds to the $\mathrm{F}$ box protein Skp2 and inhibits the ubiquitination and proteasomal degradation of c-Myc. FEBS Lett 580: 431-436, 2006.

39. Zimber-Strobl U and Strobl LJ: EBNA2 and Notch signalling in Epstein-Barr virus mediated immortalization of B lymphocytes. Semin Cancer Biol 11: 423-434, 2001.

40. Weijzen S, Zlobin A, Braid M, Miele L and Kast WM: HPV16 E6 and E7 oncoproteins regulate Notch-1 expression and cooperate to induce transformation. J Cell Physiol 194: 356-362, 2003. 